\title{
$N$-th Order Ergodic Multigram HMM for Modeling of Languages without Marked Word Boundaries
}

\author{
Hubert Hin-Cheung LAW \\ Dept. of Computer Science \\ The University of Hong Kong \\ hhclaw@cs.hku.hk
}

\author{
Chorkin CHAN \\ Dept. of Computer Science \\ The University of Hong Kong \\ cchan@cs.hku.hk
}

\begin{abstract}
Hirgodic IIMMs have been successfully used for modeling sentence production. However for some oriental languages such as Chinese, a word can consist of multiple characters without word boundary markers between adjacent words in a sentence. This makes wordsegmentation on the training and testing data necessary before ergodic HMM can be applied as the language model. This paper introduces the $N$-th order Ergodic Multigram HMM for language modeling of such languages. Each state of the IIMM can generate a variable number of characters corresponding to one word. The model can be trained without wordsegmented and tagged corpus, and both segmentation and tagging are trained in onc single model. Results on its application on a Chinese corpus are reported.
\end{abstract}

\section{Motivation}

Statistical language modeling offers advantages including minimal domain specific knowledge and hand-written rules, trainability and scalability given a language corpus. Languagc models, such as $N$-gram class models (Brown et al., 1992) and Frgodic Hidden Markov Models (Kuhn et al., 1994) were proposed and used in applications such as syntactic class (POS) tagging for English (Cutting et al., 1992), clustering and scoring of recognizcr sentence hypotheses.

However, in Chinese and many other oriental languages, there are no boundary markers, such as space, between words. Therefore preprocessors have to be used to perform word segmentation in order to identify individual words before applying these word-based language rrodels. As a result current approaches to modeling these languages arc separated into two seperated processes.
Word scgmentation is by no means a trivial process, since ambiguity often cxists. For proper segmentation of a sentence, some linguistic information of the sentence should be used. However, commonly used heuristics or statistical based approaches, such as maximal matching, frequency counts or mutual information statistics, have to perform the segmentation without knowledge such as the resulting word categorics.

To reduce the impact of erroneous segmentation on the subsequent language model, (Chang and Chan, 1993) used an $N$-best segmentation interface between them. However, since this is still a two stage model, the parameters of the whole model cannot be optimized together, and an $\mathrm{N}$ best interface is inadequate for processing outputs from recognizers which can be highly ambiguous.

$\Lambda$ better approach is to kecp all possible segmentations in a lattice form, score the lattice with a language model, and finally retrieve the best candidate by dynamic programming or some searching algorithms. N-gram models are usually used for scoring (Gu et al., 1991) (Nagata, 1994), but their training requires the sentences of the corpus to be manually segmented, and even class-tagged if class-based $N$-gram is used, as in (Nagata, 1994).

A language model which considers segmentation ambiguities and integrates this with a $N$ gram model, and able to be trained and tested on a raw, unsegmented and untagged corpus, is highly desirable for processing languages without marked word boundaries.

\section{The Ergodic Multigram HMM Model}

\subsection{Overview}

Based on the Hidden Markov Model, the Hrgodic Multigram Hidden Markov Model (Law and Chan, 1996), when applied as a language model, can process directly on unsegmented input corpus 
as it allows a variable number of characters in cach word class. Other than that its properties are similar to Frgodic Hidden Markov Models (Kuhn et al., 1994), that both training and scoring can be done directly on a raw, untagged corpus, given a lexicon with word classes.

Specifically, the $N$-th order brgodic Multigram II MM, as in conventional class-based $(N+1)$-gram model, assumes a doubly stochastic process in sentence production. The word-class sequence in a sentence follows the $N$-th order Markov assumption, i.e. the identity of a class in the sentence depends only on the previous $N$ classes, and the? word observed depends only on the class it belongs to. The difference is that this is a multigram model (Deligne and Bimbot, 1995) in the sense that cach state (i.e. node in the II MM) can generate a variable number of observed character sequences. Sentence boundaries are modeled as a special class.

This model can be applied to an input sentence or a character lattice as a language model. 'The maximum likelihood state sequence through the model, obtained using the Viterbi or Stack becoding $\Lambda$ gorithm, represents the best particular segmentation and class-tagging for the input sentence or latitice, since transition of states denotes a word boundary and state identity denotes the current word class.

\subsection{Lexicon}

A lexicon (CKIP, 1993) of 78,322 words, cach containing up to 10 characters, is available for use in this work. Practically all characters have an entry in the lexicon, so that out-of-vocabulary words aro modeled as individual characters. 'Ihere is a total of 192 syntactic classes, arranged in a hicrarchical way. For example, the month names are denoted by the class Ndabc, where $\mathrm{N}$ denotes Noun, Nd denotes 'T'mporal Nouns, Nda for 'l'ime names and Ndab for reusable time names. 'l'here is a total of 8 major categories.

liach word in the dictionary is amotated with one or more syntactic tags, representing diflerent syntaclic classes the word can possibly belong to. Also, a frequency count for ach word, based on a certain corpus, is given, but without information on its distribution over different syntactic chasses.

\subsection{Terminology}

Let $\mathcal{W}$ be the set of all Chincse words in the lexicon. $\Lambda$ word $w_{k} \in \mathcal{W}$ is made up of one or more characters. Let $s_{1}^{T}=\left(s_{1}, s_{2}, \ldots s_{T}\right)$ denote a sentence as a $T$-character sequence. $\Lambda$ function $\delta_{w}$ is defined such that $\delta_{w}\left(w_{k}, s_{t}^{t+r-1}\right)$ is 1 if $w_{k}$ is a $r$-character word $s_{t} \ldots s_{t+r-1}$, and 0 otherwise. ${ }^{1}$ let, $R$ be the upper bound of $r$, i.e. the maximum number of characters in a word (10 in this paper).

Let $C=\left\{c_{1} \ldots c_{2}\right\}$ be the set of syntactic classes, whore $I$, is the number of syntactic classes in the lexicon (192 in our case). Iot $\mathcal{C} \subset \mathcal{W} \times C$ denote the relation for all syntactic classifications of the lexicon, such that $\left(w_{k}, c_{l}\right) \in \mathcal{C}$ iff $c_{l}$ is one of the syntactic classes for $w_{k}$. Wach word $w_{k}$ mulst belong to one or more of the classes.

$\Lambda$ path through the model represents a particnlar segmentation and class tagging for the sentence. Iet $\mathcal{L}=\left(w_{1}, c_{1} ; \ldots ; w_{K}, c_{K}\right)$ be a particular segmentation and class tagging for the sentence $s_{1}^{T}$, where $w_{k}$ is the $k$ th word and $c_{k}$ denotes the class assigned to $w_{k}$, as illustrated bolow.

$$
(\overbrace{s_{1} \ldots s_{l_{1}-1}}^{w_{1}, c_{l_{1}}} \ldots \overbrace{s_{l_{k-1}} \ldots s_{l_{k}-1}}^{w_{l_{k}}, c_{l_{k}}} \ldots \overbrace{s_{l_{K-1}} \ldots s_{s_{T}}}^{w_{K}, c_{l_{K}}})
$$

lior $\mathcal{L}$ to be proper, $\prod_{k=1}^{K} \delta_{w}\left(w_{k}, s_{k_{k-1}}^{t_{k}-1}\right)=1$ and $\left(w_{k}, c_{l_{k}}\right) \in \mathcal{C}$ must be satisfied, where $t_{0}=1, t_{K}=$ $I^{\prime}+1$ and $t_{k-1}<t_{k}$ for $1 \leq k \leq K$.

\subsection{HMM Staties for the $N$-th order model}

In the first order IIMM (class bigram) model, each IIMM state corresponds directly to the word-class of a word. 13ut in general, for an $N$-th order IIMM model, since cach class depends on $N$ previous classes, each statio has to represent the combination of the classes of the most recent $N$ words, inclucling the current word.

Let $Q_{i}$ represent a state of tho $N$-th order lirgodic Multigran II MM. 'Thus $Q_{i}=\left(c_{i_{0}} \ldots c_{i_{N-1}}\right)$ where $c_{i_{0}}$ is the current word class, $c_{i_{1}}$ is the previous word class, etc. There is a total of $J^{N}$ states, which may moan too many paraneters ( $I^{N+1}$ possible state transitions, each state can transit to $l$, other states) for the model if $N$ is anything greater than onc.

'lo solve this problem, il reasonable assumption can be made that, the detailed class identities of a more distant word lave, in general, less influence than the closer ones to the current word class. 'I'hus instead of using $\mathcal{C}$ as the classification relation for all previous words, a set of

\footnotetext{
"The algorithm to be described assumes that the character identities are known for the sentence $s_{1}^{r}$, but it can also be applied when each character position se becomes a set of possible character candiclates by simply letting $\delta_{w}\left(w_{k}, s_{t}^{t+r-1}\right)=1$ for all words $w_{k}$ which can be constructed from the character positions $s_{t} \ldots s_{t+r-1}$ of the input claraster lattice. This enables the model to be used as the language model component for recognizers and for decoding phonetic: input.
} 
classification relations $\left\{\mathcal{C}^{(0)}, \mathcal{C}^{(1)}, \ldots \mathcal{C}^{(N-1)}\right\}$ can be used, where $\mathcal{C}^{(0)}=\mathcal{C}$ represents the original, most detailed classification relation for the current word, and $\mathcal{C}^{(n)}$ is the less detailed classification scheme for the $n$th previous word at each state. Thus the number of states reduces to $L_{Q}=L^{(0)} L^{(1)} \ldots L^{(N-1)}$ in which $L^{(n)} \leq L$. Each state is represented as $Q_{i}=\left(c_{i_{0}}^{(0)} \ldots c_{i_{N-1}}^{(N-1)}\right)$ where $C^{(n)}=\left\{c_{l}^{(n)}\right\}, 1 \leq l \leq L^{(n)}$ is the class tag set for the $n$th previous word.

However, if no constraints are imposed on the series of classification relations $\mathcal{C}^{(n)}$, the number of possible transitions may increase despite a decrease in the number of states, since state transitions may become possible between every two state, resulting in a total of $L^{(0)^{2}} L^{(1)^{2}} \ldots L^{(N-1)^{2}}$ possible transitions.

A constraint is imposed that, given that a word belongs to the class $c_{l}^{(n)}$ in the classification $\mathcal{C}^{(n)}$, we can determine the corresponding word class $c_{\eta^{\prime}}^{(n+1)}$ the given word will belong to in $\mathcal{C}^{(n+1)}$, and for every word there is no extra classifications in $\mathcal{C}^{(n+1)}$ not corresponding to one in $\mathcal{C}^{(n)}$. Formally, there exist mapping functions $\mathcal{F}^{(n)}$ : $C^{(n)} \mapsto C^{(n+1)}, 0 \leq n \leq N-2$, such that if $\left(c_{l}^{(n)}, c_{l^{\prime}}^{(n+1)}\right) \in \mathcal{F}^{(n)}$, then $\left(\left(w_{k}, c_{l}^{(n)}\right) \in \mathcal{C}^{(n)}\right) \Rightarrow$ $\left(\left(w_{k}, c_{\eta^{\prime}}^{(n+1)}\right) \in \mathcal{C}^{(n+1)}\right)$ for all $w_{k} \in \mathcal{W}$, and that $\mathcal{F}^{(n)}$ is surjective. In particular, to model sentence boundaries, we allow $\$$ to be a valid class tag for all $C^{(n)}$, and define $\mathcal{F}^{(n)}(\$)=\$$.

The above constraint ensures that given a state

$$
Q_{i}=\left(c_{i_{0}}^{(0)} \ldots c_{i_{N-1}}^{(N-1)}\right)
$$

it can only transit to

$$
Q_{j}=\left(c_{j_{0}}^{(0)}, \mathcal{F}^{(0)}\left(c_{i_{0}}^{(0)}\right) \ldots \mathcal{F}^{(N-2)}\left(c_{i_{N-2}}^{(N-2)}\right)\right)
$$

where $c_{j_{0}}^{(0)}$ is any state in $C^{(0)}$. Thus reducing to the maximum number of possible transitions to $L^{(0)^{2}} L^{(1)} \ldots L^{(N-1)}$.

This constraint is easily satisfied by using a hierarchical word-class scheme, such as the one in the CKIP lexicon or one generated by hierarchical word-clustering, so that the classification for more distant words (higher $n$ in $\mathcal{C}^{(n)}$ ) uses a higher level, less detail tag set in the scheme.

\subsection{Sentence Likelihood Formulation}

Let $\{\mathcal{L}\}$ be the set of all possible segmentations and class taggings of a sentence. Under the $N$ th order model $\Theta^{N}$, the likelihood of each valid segmentation and tagging $\mathcal{C}$ of the sentence $s_{1}^{T}$, $P\left(s_{1}^{T}, \mathcal{L} \mid \Theta^{N}\right)$, can be derived as follows.

$$
P\left(w_{1}, c_{l_{1}} ; w_{2}, c_{2} ; \ldots ; w_{K}, c_{l_{K}} \mid \Theta^{N}\right)
$$

$$
\begin{aligned}
= & P\left(w_{1} \mid c_{l_{1}}\right) P\left(c_{l_{1}} \mid \$^{N}\right) P\left(\$ \mid c_{l_{K}} \ldots c_{l_{K-N+1}}\right) \times \\
& \left(\prod_{k=2}^{K} P\left(w_{k} \mid c_{l_{k}}\right) P\left(c_{l_{k}} \mid c_{l_{k-1}} \ldots c_{l_{k-N}}\right)\right) \\
= & P\left(w_{1} \mid c_{l_{1}}\right) P\left(Q_{l_{1}} \$^{N}\right) P\left(\$ \mid Q_{l_{K}}\right) \times \\
& \left(\prod_{k=2}^{K} P\left(w_{k} \mid c_{l_{k}}\right) P\left(Q_{l_{k}} \mid Q_{l_{k-1}}\right)\right)
\end{aligned}
$$

using $N$ th order Markov assumption and representing the class history as HMM states. \$ denotes the sentence boundary, $c_{l_{k}}$ is $\$$ for $k \leq 0$, and

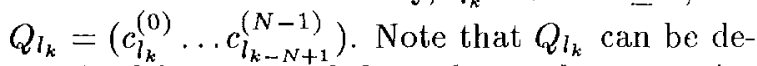
termined from $c_{l_{k}}$ and $Q_{l_{k-1}}$ due to the constraint on the classification, and thus $P\left(Q_{l_{k}} \mid Q_{l_{k-1}}\right)=$ $P\left(c_{l_{k}} \mid Q_{l_{k-1}}\right)$.

The likelihood of the sentence $s_{1}^{T}$ under the model is given by the sum of the likelihoods of its possible segmentations.

$$
P\left(s_{1}^{T} \mid \Theta^{N}\right)=\sum_{\mathcal{L} \in\{\mathcal{L}\}} P\left(s_{1}^{T}, \mathcal{L} \mid \Theta^{N}\right)
$$

\section{The Algorithms}

\subsection{The Parameters}

As in conventional HMM, the Ergodic Multigram HMM consists of parameters $\Theta^{N}=\{A, B\}$, in which $A=\left\{a_{i j}\right\}, 0 \leq i, j \leq I_{Q}$ (Total number of states), denotes the set of state transition probabilities from $Q_{i}$ to $Q_{j}$, i.e. $P\left(Q_{j} \mid Q_{i}\right)$. In particular, $a_{0 i}=P\left(Q_{i} \mid \$^{N}\right)$ and $a_{i 0}=P\left(\$ \mid Q_{i}\right)$ denote the probabilities that the state $Q_{i}$ is the initial and final state in traversing the HMM, respectively. $a_{00}$ is left undefined. $B=\left\{b_{j}\left(w_{k}\right)\right\}$, where $1 \leq j \leq L^{Q}$, denotes the set of word observation probabilities of $w_{k}$ at the state $Q_{j}$, i.e. $P\left(w_{k} \mid Q_{j}\right)$.

The $B$ matrix, as shown above, models the probabilities that $w_{k}$ is observed given $N$ most recent classes, and contains $L_{Q}|\mathcal{W}|$ parameters (recall that $\left.L_{Q}=L^{(0)} L^{(1)} \ldots L^{(N-1)}\right)$. Our assumption that $w_{k}$ only depends on the current class reduces the number of parameters to $L^{(0)}|\mathcal{W}|$ for the $B$ matrix. 'Thus in the model, $b_{j}\left(w_{k}\right)$ representing $P\left(w_{k} \mid Q_{j}\right)$ are tied together for all states $Q_{j}$ with the same current word-class, i.c. $P\left(w_{k} \mid Q_{j}\right)=P\left(w_{k} \mid c_{l}\right)$ if $Q_{j}=\left(c_{l} \ldots\right)$. Also, $a_{i j}$ is 0 if $Q_{i}$ cannot transit to $Q_{j}$. As a result the number of parameters in the $A$ matrix is only $I^{(0)} L_{Q}$.

Given the segmentation and class sequence $\mathcal{L}$ of a sentence, the state sequence $\left(Q_{I_{1}} \ldots Q_{l_{k}}\right)$ can be derived from the class sequence $\left(c_{l_{1}} \ldots c_{l_{k}}\right)$. Thus the observation probability of the sentence $s_{1}^{T}$ given $\mathcal{L}$ and the model $\Theta^{N}, P\left(s_{1}^{T}, \mathcal{L} \mid \Theta^{N}\right)$, can be reformulated as

$$
b_{l_{1}}\left(w_{1}\right) a_{0 l_{1}} a_{l_{K} 0}\left(\prod_{k=2}^{K} a_{l_{k-1} l_{k}} b_{l_{k}}\left(w_{k}\right)\right)
$$


Given this formulation the training procedure is mostly similar to that of the first order Ergodic Multigram HMM.

\subsection{Forward and Backward Procedure}

The forward variable is defined as

$$
\alpha_{t}(i)=P\left(s_{1} \ldots s_{t}, Q_{l(t)}=Q_{i} \mid \Theta^{N}\right)
$$

where $Q_{l(t)}$ is the state of the IIMM when the word containing the character $s_{l}$ as the last character is produced.

The recursive equations for $\alpha_{t}(i)$ aro

$$
\begin{aligned}
\alpha_{t}(j)= & 0 \text { for } t<1 \\
\alpha_{t}(j)= & \sum_{w_{k} \in \mathcal{W}} a_{0 j} b_{j}\left(w_{k}\right) \delta_{w}\left(w_{k}, s_{1}^{t}\right)+ \\
& \sum_{r=1}^{R} \sum_{w_{k} \in \mathcal{W}}\left[\sum_{i=1}^{L_{Q}} \alpha_{t-r}(i) a_{i j} b_{j}\left(w_{k}\right)\right] \\
& \delta_{w}\left(w_{k}, s_{t-r+1}^{t}\right) \\
& \text { for } 1 \leq t \leq T^{\prime}
\end{aligned}
$$

Similarly, the backward variable is defined as

$$
\beta_{t}(i)=P\left(s_{t+1} \ldots s_{T} \mid Q_{l(t)}=Q_{i}, \Theta^{N}\right)
$$

'The recursive equations for $\beta_{t}(i)$ are

$$
\begin{aligned}
\beta_{t}(i)= & 0 \text { for } t>T \\
\beta_{T}(i)= & a_{i 0} \\
\beta_{t}(i)= & \sum_{r=1}^{n} \sum_{w_{k} \in \mathcal{W}}\left[\sum_{j=1}^{L_{Q}} a_{i j} \beta_{t+r}(j) b_{j}\left(w_{k}\right)\right] \\
& \delta_{w}\left(w_{k}, s_{t+1}^{t+r}\right) \\
& \text { for } 1 \leq t \leq T \cdots 1
\end{aligned}
$$

As $A, B$ arrays and the $\delta_{u}$ function are mostly 0s, considerable simplification can be done in implementation.

'The likelihood of the sentence given the model can be evaluated as

$$
P\left(s_{1}^{T^{\prime}} \mid \Theta^{N}\right)=\sum_{i=1}^{L_{Q}} \alpha_{T}(i) a_{i 0}
$$

I'he Viterbi algorithm for this nodel can be ob tained by replacing the summations of the forward algorithm with maximizations.

\subsection{Re-estimation Algorithm}

$\xi_{t}(i, j)$ is defined as the probability that given a sentence $s_{1}^{\prime}$ and the model $\Theta^{N}$, a word ends at the character $s_{t}$ in the state $Q_{i}$ and the next word starts at the character $s_{t+1}$ in the state $Q_{j}$. 'Thus $\xi_{t}(i, j)$ can be expressed as

$$
\frac{\sum_{r=1}^{R} \sum_{w_{k} \in \mathcal{W}} \alpha_{t}(i) a_{i j} b_{j}\left(w_{k}\right) \delta_{u}\left(w_{k}, s_{t+1}^{t+r}\right) \beta_{t+r}(j)}{P\left(s_{1}^{T} \mid \Theta^{N}\right)}
$$

for $1 \leq t \leq I^{\prime}-1,1 \leq i, j \leq L_{Q}$. Furthermore define $\gamma_{t}(i)$ to be the probability that, given $s_{1}^{T}$ and $\Theta^{N}$, a word ends at the character $s_{t}$ in the state $Q_{i}$. 'T'hus

$$
\gamma_{t}(i)=\frac{\alpha_{t}(i) \beta_{t}(i)}{P\left(s_{1}^{T} \mid \Theta^{N}\right)} \text { for } 1 \leq t \leq T, 1 \leq i \leq L_{Q}
$$

Summation of $\xi_{t}(i, j)$ over $t$ gives the expected number of times that state $Q_{i}$ transits to state $Q_{j}$ in the sentence, and summation of $\gamma_{t}(i)$ over $t$ gives the expected number of state $Q_{i}$ occurring' in it. 'I'hus the quotient of their summation over $t$ gives $a_{i j}$, the new estimation for $a_{i j}$.

$$
a_{i j}=\sum_{t=1}^{T-1} \xi_{t}(i, j) / \sum_{t=1}^{T} \gamma_{t}(i) \text { for } 1 \leq i, j \leq L_{Q}
$$

The initial and fmal class probability estimates, $\bar{a}_{0 j}$ and $\ddot{a}_{i 0}$ can be re-estimated as follows.

$$
\begin{aligned}
a_{0 j}= & \frac{\sum_{r=1}^{R} \sum_{w_{k} \in \mathcal{W}} a_{0 j} b_{j}\left(w_{k}\right) \delta_{w}\left(w_{k}, s_{1}^{r}\right) \beta_{r}(j)}{P\left(s_{1}^{T^{\prime}} \mid \Theta^{N}\right)} \\
a_{i 0}= & \frac{\alpha_{T}^{\prime}(i) a_{i 0}}{P\left(s_{1}^{T^{\prime}} \mid \Theta^{N}\right)} / \sum_{t=1}^{T} \gamma_{t}(i)
\end{aligned}
$$

Io derive $b_{j}\left(w_{k}\right)$, first define $\alpha_{t}^{w_{k}}(i)$ as the protability of the sentence prefix $\left(s_{1} \ldots s_{t}\right)$ with $w_{k}$ in state: $Q_{i}$ as the last complete word. 'I'hus

$$
\begin{aligned}
\alpha_{t}^{w_{k}}(j)= & a_{0 j} b_{j}\left(w_{k}\right) \delta_{w}\left(w_{k}, s_{1}^{t}\right)+\sum_{r=1}^{R} \sum_{i=1}^{L_{Q}} \\
& \left(\alpha_{t-r}(i) a_{i j} b_{j}\left(w_{k}\right) \delta_{w}\left(w_{k}, s_{t-r+1}^{t}\right)\right)
\end{aligned}
$$

'I'his represents the contribution of $w_{k}$, occurring as the last word in $s_{1}^{t}$, to $\alpha_{t}(j)$. Also define $\gamma_{t}^{i w_{k}}(j)$ to be the probability that, given the sentence $s_{1}^{T}$ and the model, $w_{k}$ is observed to ond at character $s_{t}$ in the state $Q_{j}$.

$$
\gamma_{t}^{w_{k}}(j)=\frac{\alpha_{t}^{w_{k}}(j) \beta_{t}(j)}{P\left(s_{1}^{T} \mid \Theta^{N}\right)}
$$

Let $Q_{j} \diamond Q_{j}$ denotes the relation that both $Q_{j}$ and $Q_{j}$ represent the same current word class. 'Thus summation of $\gamma_{t}^{w_{k}}(j)$ over $t$ gives the cxpected number of times that $w_{k}$ is observed in 
state $Q_{j}$, and summation of $\gamma_{t}(j)$ over $t$ gives the total expected number of occurrence of state $Q_{j}$. Since states with the same current word class are tied together by our assumption, the required value of $\bar{b}_{j}\left(w_{k}\right)$ is given by

$$
\tilde{b}_{j}\left(w_{k}\right)=\frac{\sum_{\substack{Q_{j} \diamond Q_{j^{\prime}} \\ j^{\prime}}} \sum_{t=1}^{T} \gamma_{t}^{w_{k}}\left(j^{\prime}\right)}{\sum_{Q_{j} \diamond Q_{j^{\prime}}}^{j^{\prime}} \sum_{t=1}^{T} \gamma_{t}\left(j^{\prime}\right)}
$$

\section{Experimental Results}

\subsection{Setup}

A corpus of daily newspaper articles is divided into training and testing sets for the expcriments, which is $21 \mathrm{M}$ and $4 \mathrm{M}$ in size respectively. The first order $(N=1)$ algorithms are applied to the training sets, and parameters obtained after different iterations are used for testing.

The initial parameters of the HMM are set based on the frequency counts from the lexicon. The class-transition probability $a_{i j}$ is initialized as the a priori probability of the state $P\left(Q_{j}\right)$, estimated from the relative frequency counts of the lexicon. $b_{j}\left(w_{k}\right)$ is initialized as the relative count of the word $w_{k}$ within the class corresponding to the current word class in $Q_{j}$. Words belonging to multiple classes have their counts distributed equally among them. Smoothing is then applied by adding each word count by 0.5 and normalizing.

After training, the Viterbi algorithm is used to retrieve the best segmentation and tagging $\mathcal{L}^{*}$ of each sentence of the test corpus, by tracing the best state sequence traversed.

\subsection{Perplexity}

The test-sct perplexity, calculated as

$$
P P^{*}=\exp \left(-\frac{1}{M} \sum_{i} \log \left(I^{\prime}\left(s_{1}^{T_{i}}, \mathcal{L}^{*} \mid \Theta^{N}\right)\right)\right)
$$

where the summation is taken over all sentences $s_{1}^{T_{i}}$ in the testing corpus, and $M$ represents the number of characters in it, is used to measure the performance of the model.

'The results for models trained on training corpus subsets of various sizes, and after various iterations are shown ('Table 1). It is obvious that with small training corpus, over-training occurs with morc iterations. With more training data, the performance improves and over-training is not cvident.

\subsection{Phonetic Input Decoding}

A further experiment is performed to use the models to decode phonetic inputs (Gu et al., 1991).

\begin{tabular}{|c||cccc|}
\hline Training Size & 2 & 4 & 6 & 8 \\
\hline $98 \mathrm{~K}$ & 194.009 & 214.096 & 246.613 & 286.721 \\
$1.3 \mathrm{M}$ & 126.084 & 122.304 & 121.606 & 121.776 \\
$6.3 \mathrm{M}$ & 118.531 & 113.600 & 111.745 & 110.783 \\
$21 \mathrm{M}$ & 116.376 & 111.275 & 109.282 & 108.142 \\
\hline
\end{tabular}

Table 1: 'Test Set Perplexities of testing set after different iterations on subsets of training set

'Lhis is not trivial since each Chinese syllable can correspond to up to 80 different characters. Sentences from the testing corpus are first expanded into a lattice, formed by generating all the cormmon homophones of each Chinese character. 'Tested on $360 \mathrm{~K}$ characters, a character recognition rate of $91.24 \%$ is obtained for the model trained after 8 iterations with $21 \mathrm{M}$ of training text. The results are satisfactory given that the test corpus contains many personal names and out of vocabulary words, and the highly ambiguous nature of the problem.

\section{Discussion and Conclusion}

In this paper the $N$-th order Ergodic Multigram IIMM is introduced, whose application enables integrated, itcrative language model training on untagged and unsegmented corpus in languages such as Chinese.

The performance on higher order models are expected to be better as the size of training corpus is relatively large. However some form of smoothing may have to be applied when the training corpus size is small.

With some modification this algorithm would work on phoneme candidate input instead of character candidate input. This is useful in decoding phonetic strings without character boundaries, such as in continuous Chinese/Japanese/Korean phonetic input, or speech recognizers which output phonemes.

This model also makes a wealth of techniques developed for IIMM in the specch recognition ficld available for language modeling in thesc languages.

\section{References}

Brown, P.F., deSouza, P.V., Mercer, R.L., Della Pietra, V.J., Lai, J.C. 1992. Class-Based $n$ gram Models of Natural Language. In Computational Linguistics, 18:167-479.

Chang, C.II., Chan, C.I. 1993. A Sudy on Integrating Chinese Word Segmentation and l'art- 
of-Speech 'Tagging. In Comm. of COLHPS' Vol 3, No.1, pp.69-77.

Chinese Knowledge Information Group 1993. In Technical Report No. 93-05. Institute of Information Science, Academia Sinica, 'Taiwan.

Cutting, K., Kupiec, J., Pedersen, J., Sibun, P. 1992. A Practical Part-of-Spech Tagger. In Proceedings of the Third Conference on Applicd Natural Language Processing, Ip. 133-140.

Deligno, S., Bimbot, F. 1995. Language Modeling by Variable Iength Sequences: I'heoretical Formulation and Lvaluation of Multigrams. In ICASSP 95, pI.169-172.

Gu, II.Y., 'I'seng, C.Y., Lee, I..S. 1991. Markov Modeling of Mandarin Chinese for decoling the phonetic sequence into Chinese chatacters. In Compuler spech and language, Vol 5, pp.303\%377.

Kuhn, T', Nicmann, H., Schukat - Talamaz'ini, F.G. 1994. Hrgodic Hidden Markov Modcls and Polygrams for Ianguage Modeling. In ICASSP 94, pp.357-360.

Law, II.II.C., Chan, C. 1996. Frgodic Multigram IIMM Integrating Word Segmentation and C'lass 'Iagging for Chinese Language. Modcling. 'To appeas' in ICASSP' 96.

Nagata, M. 1994. A Stochastic Japanese Morphological Analyzer Using a lorward-1)p Backward- $\Lambda^{*} N$-Best Search Algorithm. In COIING 94, pp.201-207. 